\title{
Cumulative readings beyond nominals
}

\author{
Patrick D. Elliott, Andreea C. Nicolae*
}

\begin{abstract}
In this paper, our aim is to investigate the limits of semantic plurality; we ask whether semantic plurality constrained by morphosyntactic plurality, and if so, how. In order to shed light on these questions, we focus on two empirical domains of inquiry in which we find superficial mismatches between semantic and morphosyntactic plurality: (i) group nouns and agreement in British vs. English, and (ii) semantically plural embedded interrogatives. Based on our findings, we argue for a new, negative licensing condition on the insertion of the $*$ and $* *$ operators.
\end{abstract}

Keywords. semantics; morphosyntax; questions; plurality; distributivity; cumulativity; number

1. Overview. In $\S 2$, we provide background on the system assumed here for analyzing semantic plurality, which builds largely on work by Winter (2001). In $\S 3$, we introduce two logically distinct routes to distributive inferences with plural subjects: phrasal distributivity and lexical distributivity, and summarize arguments that different routes must be made available by the grammar, building on de Vries (2015). In $\S 4$ we move towards a unified picture of plurality effects by arguing for the availability of phrasal cumulativity and lexical cumulativity, subject to the same constraints. In $\S 5$ we turn to questions and discuss how plurality manifests itself in that domain with respect to distributive and cumulative inferences. We conclude that semantic plurality is domain general in that we observe phrasal distributivity and cumulativity with plural interrogatives, and, syntactically constrained, in that since phrasal distributivity and cumulativity seem to be parasitic on morphosyntactic plurality.

\section{Background.}

Pluralization In the (substantial) existing literature on semantic plurality, there is a general split according to whether pluralities are modelled as sets (see, e.g., Schwarzschild 1996; Winter 2001; Champollion 2015; de Vries 2015, etc.) or i-sums (Link 1983; Landman 1996 and much subsequent work). In this work, we adopt the former view. Despite the fact that the two approaches are to a large extent isomorphic ${ }^{1}$, adopting the view of pluralities as sets will allow us to give an account of the domain generality of semantic plurality by invoking set-theoretic operations. We therefore take singular NPs to denote sets of atomic individuals, and plural NPs to denote sets of sets of atomic individuals, i.e., a set of pluralities, as illustrated in (1) and (2) respectively. ${ }^{2}$

$$
\text { (1) } \llbracket \text { poet } \rrbracket=\{\text { Rilke, Yeats, Elliot }\}
$$

${ }^{*}$ We would like to thank the audience at the 2016 Annual Meeting of the Linguistics Association of Great Britain, and attendees at the Linguistic Society of America 2017 Annual Meeting. The second author would also like to thank the German Federal Ministry of Research (BMBF Grant Nr. 01UG1411) for its financial support. Authors: Patrick D. Elliott, University College London (p.elliott@ucl.ac.uk) \& Andreea C. Nicolae, Leibniz-Zentrum Allgemeine Sprachwissenschaft (nicolae@leibniz-zas.de).

${ }^{1}$ Where the two theories differ is whether or not nested pluralities (i.e., pluralities of pluralities) are predicted to be possible. i-sums are flat, and therefore do not allow for such structure, whereas sets are not, and therefore predict nested pluralities to potentially be in the model. This question is largely orthogonal to our interests here, but see, e.g., Schwarzschild (1996) for discussion.

2 Throughout this article, we shift freely between set-talk and function-talk. The set theoretic operations we invoke, such as powerset and union, can easily be redefined as operations on characteristic functions. 


$$
\llbracket \text { poets } \rrbracket=\left\{\begin{array}{c}
\{\text { Rilke }\},\{\text { Yeats }\},\{\text { Elliot }\}, \\
\{\text { Rilke, } \text { Yeats }\},\{\text { Rilke }, \text { Elliot }\},\{\text { Yeats, Elliot }\}, \\
\{\text { Rilke, Yeats, Elliot }\}
\end{array}\right\}
$$

Following, e.g., Winter (2001) and de Vries (2015), we take the plural morpheme PL to introduce the $*$-operator. $*$ is defined as an operator which takes a set, and returns its powerset minus the empty set, as in (3). Note that we assume a type-flexible denotation for $*$.

(3) Predicate Pluralization (after de Vries 2015, ex. 2, pp. 10)

$$
* P_{\langle\sigma, \mathrm{t}\rangle}:=\wp(P)-\emptyset
$$

for any type $\sigma$

Applied to our denotation for singular poet in (1), the $*$-operator returns the desired denotation for poets, i.e., the one give in (2).

$$
\llbracket \text { poets } \rrbracket=*(\llbracket \text { poet } \rrbracket)
$$

In order to account for the interpretation of singular definite descriptions as atomic individuals and plural definite descriptions as pluralities we can define a generalized definite article THE as in (5). ${ }^{3}$

(5) Generalized definite article (after de Vries 2015, ex. 4, pp. 12)

$$
\operatorname{THE}_{\langle\sigma \mathrm{t}, \sigma\rangle}\left(P_{\sigma t}\right):=\operatorname{MAX}(P)
$$

For the purposes of this work, we take DP conjunction to be interpreted as set union, as in (6), in order to account for the interpretation of conjoined DPs as pluralities, $(7 \mathrm{c}){ }^{4}$

$$
\llbracket\left[\text { andP } \mathrm{DP}_{1}\left[\text { and } \text {, and } \mathrm{DP}_{2}\right]\right] \rrbracket=\llbracket \mathrm{DP}_{1} \rrbracket \cup \llbracket \mathrm{DP}_{2} \rrbracket
$$

(7) a. $\llbracket$ the boys $\rrbracket=\{$ Abed, Troy, Jeff $\}$

b. $\llbracket$ the girls $\rrbracket=\{$ Annie, Shirley, Britta $\}$

c. 【the girls and the boys $\rrbracket=\llbracket$ the girls $\rrbracket \cup$ the boys $\rrbracket$

$$
=\{\text { Abed, Troy, Jeff, Annie, Shirley, Britta }\}
$$

In order to deal with coordinated singular DPs, we assume that the coordinands must first be typelifted into singleton sets via Partee's (1986) IDENT type-shifter, (8), delivering (9).

$$
\operatorname{IDENT}(\alpha):=\{\alpha\}
$$

$$
\begin{aligned}
\llbracket[\text { andP Rilke [and } \text { and Yeats }]] \rrbracket & =\operatorname{IDENT}(\llbracket \text { Rilke } \rrbracket) \cup \operatorname{IDENT}(\llbracket \text { Yeats } \rrbracket) \\
& =\{\text { Rilke }\} \cup\{\text { Yeats }\} \\
& =\{\text { Rilke, Yeats }\}
\end{aligned}
$$

Collective predicates Note that in the framework assumed here, there is a type distinction between singular and plural DPs: singular DPs are of type e, whereas plural DPs are of $\langle e, t\rangle$. We can marshal this distinction in order to account for the selectional restrictions of collective predicates such as to gather in a straightforward way (following Winter 2001). We assume that collective predicates require set-denoting arguments, as captured in the lexical entry in (10):

\footnotetext{
${ }^{3}$ When applied to a set of pluralities (type $\langle$ et, $t\rangle$ ), MAX returns the smallest plurality that is greater than or equal to all pluralities in the set. When applied to a set of individuals, MAX reduces to the $\iota$-operator; it will only be defined for singleton sets of individuals.

${ }^{4}$ see Winter (2001) and Champollion (2015) for an account of DP conjunction in terms of boolean coordination, which derives the same result, and is compatible with the assumptions made here.
} 


$$
\llbracket \text { gather } \rrbracket=\lambda X \in D_{\langle e, t\rangle} . X \in \text { gather }
$$

A collective predicate such as gather will thus straightforwardly compose with a plural DP such as the girls, (11a), but when it composes with a singular DP such as Annie, (11b), it will give rise to a type mismatch, delivering the incompatibility of individual-denoting nouns and collective predicates.

a. $\quad 1$ iff $\{$ Annie, Britta, Shirley $\} \in$ gather

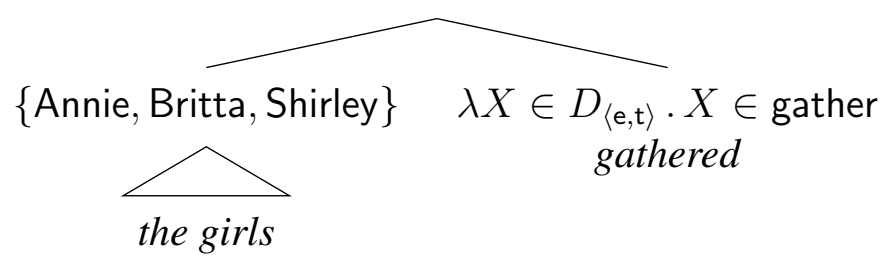

b.

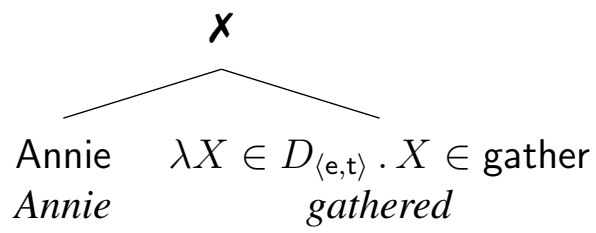

Distributive predicates We assume that distributive predicates, such as to sneeze, denote (the characteristic function of) a set of atomic individuals, as in (12).

$$
\llbracket \text { sneeze } \rrbracket=\lambda x \in D_{e} . x \in \text { sneeze }
$$

Distributive predicates are notable in that they license distributive inferences with a semantically plural argument. For example, (13) entails that each of the poets $x$ is such that $x$ sneezed.

(13) The poets sneezed.

Distributive inferences do not follow from the system assumed here without a small modification; since plural DPs are of type $\langle e, t\rangle$, and distributive predicates are also of type $\langle e, t\rangle$, a plural DP cannot compose with a distributive predicate directly, assuming that predicates and their arguments must compose via Heim \& Kratzer's (1998) rule of FunCtional ApPLiCATION (FA). We can resolve this by type-lifting the VP using the $*$-operator (parallel to the widely assumed predicate distributivity operator), which, the reader may recall, is the same operator used to introduce plural morphology in the nominal domain. We illustrate how this works in (14) (abstracting away from the internal composition of the plural definite).

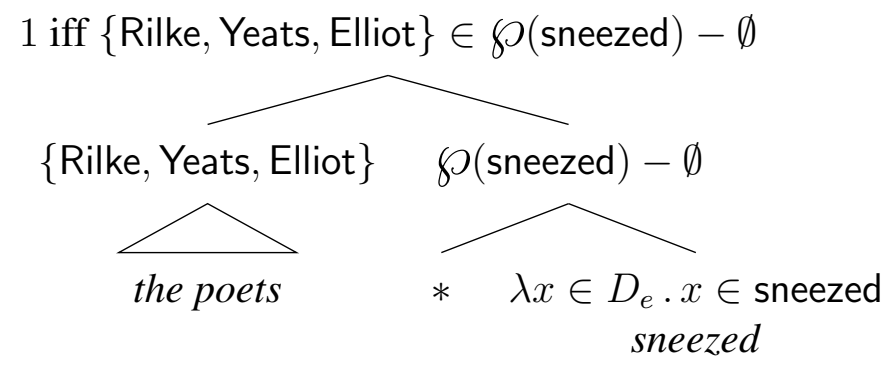

Note that (13) will only be true, according to the analysis outlined above, if the powerset includes the plurality denoted by the subject. However, by virtue of how power sets work, as soon as that 
holds, each atomic individual in the extension of the poets will also have to be in the extension of sneezed, hence the distributive inference.

In this section we showed how distributive inferences can be modeled straightforwardly by allowing plural VPs to compose with the $*$-operator. In the next section we turn to an alternative account of distributive inferences, and arguments that the $*$-operator must be necessary.

\section{Deriving distributivity.}

Lexical distributivity Invoking the *-operator is not the only way to account for distributive inferences with plurals within the framework assumed here. Scha (1981) suggests that, rather than involving an LF operator, distributive inferences arise due to the lexical semantics of distributive predicates. We can cash out Scha's view in the system assumed here by taking both collective and distributive predicates to be of type $\langle\langle e, t\rangle, t\rangle$ and building the distributive inference into the lexical semantics of the predicate itself. ${ }^{5,6}$

$$
\begin{aligned}
& \text { Scha-style entry for a distributive predicate } \\
& \text { 【to sneeze } \rrbracket=\lambda X \in D_{\langle\mathrm{e}, \mathrm{t}\rangle}: X \neq \emptyset . \forall x[x \in X \rightarrow x \in \text { sneeze }]
\end{aligned}
$$

On the face of it, the Scha-style entry in (15) and the standard entry in tandem with the $*$-operator seem to make identical predictions. Winter (2001), however, argues that one can tease them apart, and once one does, it becomes clear that the $*$-operator is necessary. We turn to this next.

Winter's (2001) argument for phrasal distributivity Scha's lexical theory of distributivity does not predict scopal interaction with other operators, whereas the $*$-operator approach potentially does. Consider the example below:

The boys are singing or dancing.

Scha's approach predicts that (16) is true iff all of the boys are dancing, or all of the boys are singing. However, (16) is also true if only some boys are singing as long as all of the others are dancing. This second reading can only be captured if we allow the $*$-operator to take scope above the disjunction, as in (17), an option not available under a Scha-style analysis of distributivity.

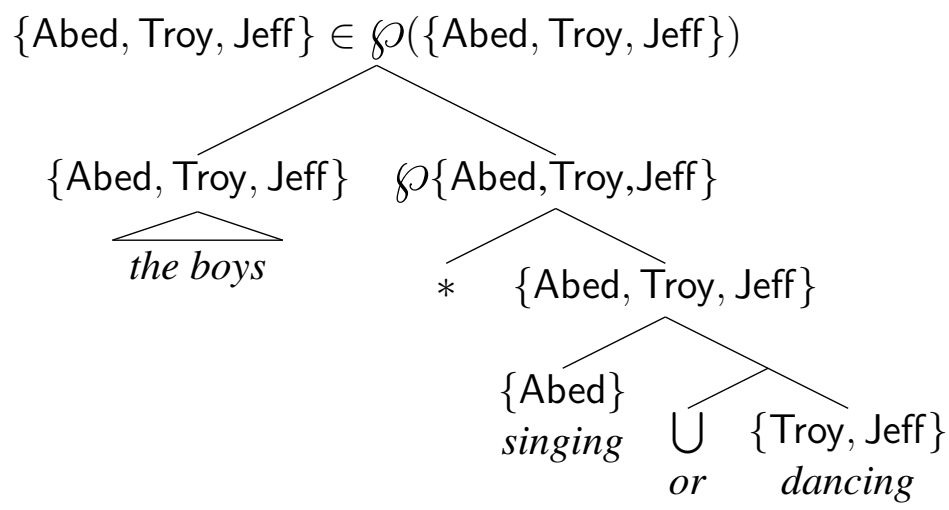

\footnotetext{
${ }^{5}$ On this view, we must invoke type-lifting in the other direction to account for the composition of singular DPs with distributive predicates. Once again, we can simply invoke Partee's IDENT type-shifter, in order to shift the atomic individual denoted by a singular DP subject to a singleton set.

${ }^{6}$ On the Scha-style analysis, we assume that distributive predicates also carry an existential presupposition. Otherwise, the truth conditions predicted would be too weak. Any non-referring singular DP would be predicted to be true of a distributive predicate.
} 
(18) illustrates that keeping the $*$-operator at the predicate level, i.e., not scoping it above the disjunction, will derive falsity in a mixed scenario like the one above. Note that this approach approximates Scha's approach which lexically encodes distributivity. Therefore we refer to the application of the $*$-operator to a terminal node as lexical distributivity, (18), and application of $*$ to a non-terminal node as phrasal distributivity, (17).

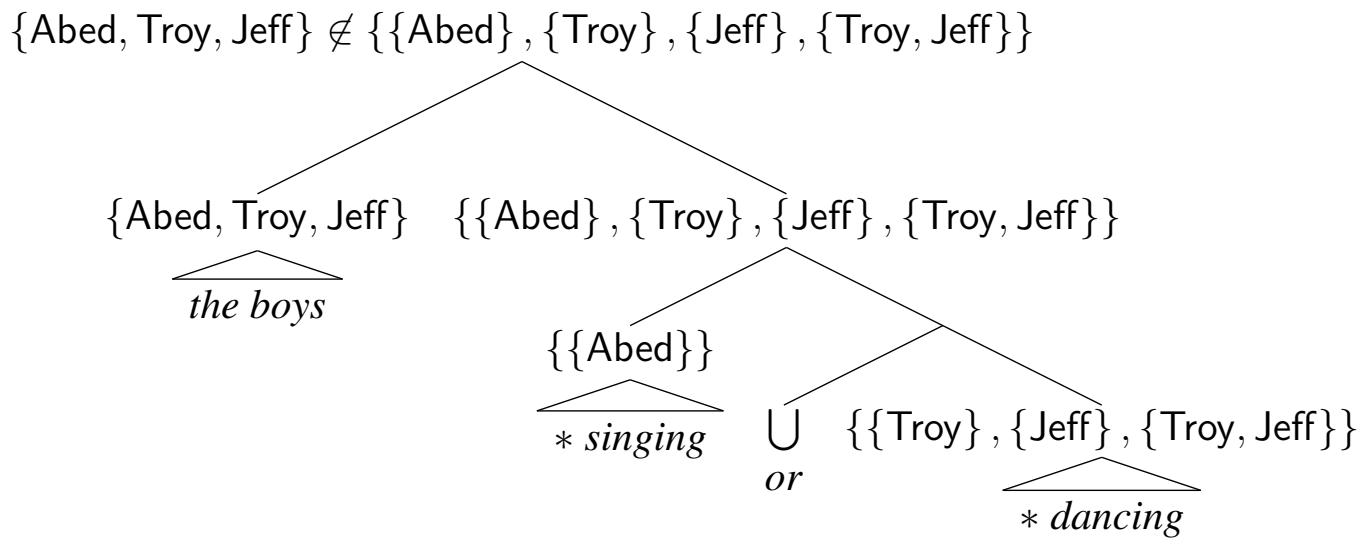

In the next section we introduce de Vries's (2015) argument that both phrasal and lexical distributivity must be made available by the grammar.

Number agreement in British vs. American English In British but not American English, singular subjects headed by group nouns such as team and committee can trigger plural agreement on the predicate. ${ }^{7}$ de Vries (2015) noticed that the choice in singular/plural morphology dictates which of two possible readings is/are available. These facts are illustrated by the minimal pair in (19). ${ }^{8}$

a. The team is hugging or kissing.

b. \%The team are hugging or kissing.

$\boldsymbol{X}$ AmEn; $\sqrt{ } \mathrm{BrEn}$

Specifically, while both the singular in (19a) and the plural in (19b) are acceptable in scenario (20a) in which either everyone on the team is hugging each other, or everyone is kissing each other, only the plural in (19b) is acceptable in the mixed scenaro (20b), where some team members are kissing each other and some team members are hugging each other. In other words, plural agreement feeds an additional reading in British English - namely the mixed reading. de Vries concludes from this that the presence of the $*$-operator, which derives the mixed reading for (19b), is dependent on a morphosyntactically plural VP.

(20) a. Scenario 1: all team members are hugging

b. Scenario 2: some are hugging and some are kissing

$$
\begin{aligned}
& (19 a)=\text { true } ;(19 b)=\text { true } \\
& (19 a)=\text { false } ;(19 b)=\text { true }
\end{aligned}
$$

\footnotetext{
${ }^{7}$ Following existing work on the semantics of group nouns (Pearson 2011), we assume that they are (or at least, have a reading according to which they are) semantically plural (Munn 1999; Elbourne 1999; Pearson 2011). In other words, we take the plural definite the team to denote the maximal set of team members (cf. Barker 1992).

${ }^{8}$ Agreement in British English with group nouns has other semantic consequences, such as bleeding the inverse scope reading in examples such as the following (Sauerland \& Elbourne 2002, ex. 30, pp. 293)

(i) a. A Northern team is likely to be in the final.

b. A Northern team are likely to be in the final.

$\checkmark \exists>$ likely; $\checkmark$ likely $>\exists$

$\checkmark \exists>$ likely; $\boldsymbol{x}$ likely $>\exists$
} 
It's worth mentioning at this point that lexical distributivity is nonetheless still necessary in order to account for the availability of distributive inferences with singular agreement elsewhere, specifically for cases involving a group noun and a distributive predicate, such as (21b).

(21) a. The committee is convening at 9am.

$\rightsquigarrow \forall x[x \in \llbracket$ the committee $\rrbracket \rightarrow x \in \llbracket$ convening at $9 \mathrm{am} \rrbracket]$

b. The committee is tired.

$\rightsquigarrow \forall x[x \in \llbracket$ the committee $\rrbracket \rightarrow x \in \llbracket$ tired $\rrbracket]$

We therefore assume that a distributive predicate such as to be tired is fundamentally a predicate ranging over individuals, of type $\langle e, t\rangle$. The predicate itself may optionally be type-lifted via $*$ to a predicate ranging over pluralities of type $\langle\langle e, t\rangle, t\rangle$. Observe that lexical distributivity cannot be dependent on morphosyntactic plurality, as illustrated by the availability of distributive inferences in (21). Phrasal distributivity, on the other hand, must be dependent on morphosyntactic plurality, as illustrated by the unavailability of the mixed reading in (19a).

4. Lexical vs. phrasal cumulativity. In this section, we suggest that a similar argument can be made for the existence of both lexical and phrasal cumulativity on the basis of number agreement with group nouns in British English. The standard theory of cumulative readings (see, e.g., Beck $\&$ Sauerland 2000) involves a $* *$-operator, which pluralizes a relation $\mathbf{R}$ of type $\langle\sigma, \iota t\rangle$, for any types $\sigma, \iota$.

$$
\left.* * R=\left\{\left\langle X_{\iota t}, Y_{\sigma t}\right\rangle: \forall x_{\iota} \in X, \exists y_{\sigma} \in Y[\langle x, y\rangle \in R] \wedge \forall y_{\sigma} \in Y, \exists x_{\iota} \in X[\langle x, y\rangle \in R\rangle\right]\right\}
$$

The same argument as in the case of mixed readings with distributive predicates can be made for cumulative readings. Specifically, the $* *$-operator is necessary in order to account for the fact that (23) is true in a mixed scenario, namely when some of the boys are hugging some of the girls and the other boys are kissing the other girls. Skipping the details of the analysis, it can be shown that only by having the $* *$-operator scope above the disjunction can we derive this reading.

(23) The boys are hugging or kissing the girls.

As before, we see morphosyntactic plurality playing a role. Only the plural version, (24b), is acceptable in a scenario where some members of the winning team are kissing some members of the losing team, and the other winners are hugging the remaining losers.

(24) a. The winning team is kissing or hugging the losing team.

b. The winning team are kissing or hugging the losing team.

Lastly, it's worth noting that lexical cumulativity is also necessary for, e.g., cases like (25), where the members of the subject and object group nouns are distributed over.

(25) The winning team is kissing the losing team.

We will henceforth use plurality inferences as a cover term for both distributive inferences and cumulative inferences. In the previous sections, we suggested that two distinct operations are necessary in order to derive the distribution of plurality inferences. Lexical plurality is necessary in order to account for plurality inferences in the absence of morphosyntactic plurality. Phrasal plurality must also be available, but, we argued, it is contingent on the presence of morphosyn- 
tactic plurality. ${ }^{9}$ Since plurality inferences are accounted for via the application of type-flexible operators, defined as abstract set-theoretic operations, we do not necessarily expect plurality to be limited to the nominal domain; in fact, we predict plurality effects to be domain general, in the sense that anything set-denoting is a potential candidate for a semantic plurality. In the next section, we look at plurality inferences outside the nominal domain - specifically, in the domain of embedded interrogatives.

\section{Plural questions.}

Background on the semantics of questions There are a number of different approaches to the semantics of questions in the literature, but one of the most prevalent is that proposed by Hamblin (1973) and later developed by Karttunen (1977): questions denote sets of answers. Here we adopt Hamblin's theory according to which constituent questions denote sets of possible answers, where answers are modelled as propositions (i.e., sets of possible worlds). ${ }^{10}$

$$
\begin{aligned}
\llbracket \text { Which boy left?』 }=\left\{p \in D_{\langle\mathrm{s}, \mathrm{t}\rangle}: \exists x\left[x \in \text { boy }_{@} \wedge p=\left\{w^{\prime} \in D_{s}: x \in \text { left }_{w^{\prime}}\right\}\right]\right\} \\
=\left\{\begin{array}{l}
\left\{w^{\prime} \in D_{s}: \text { Jeff } \in \text { left }_{w^{\prime}}\right\} \\
\left\{w^{\prime} \in D_{s}: \text { Abed } \in \text { left }_{w^{\prime}}\right\} \\
\left\{w^{\prime} \in D_{s}: \text { Troy } \in \text { left }_{w^{\prime}}\right.
\end{array}\right\}
\end{aligned}
$$

Following recent work by Fox (2012), Nicolae (2013) and Kotek (2014), we assume that multiple questions denote pluralities of questions. ${ }^{11}$ The aforementioned authors do not refer to the meaning of a multiple question explicitly as a plurality, and our use of the term here is not intended to be innocent. We will argue that multiple questions are semantically plural in the same way morphosyntactically plural NPs are (here we follow Beck \& Sharvit 2002).

$$
\begin{aligned}
& \text { «Which boy admires which girl?》 } \\
& =\left\{Q \in D_{\langle\mathrm{st}, \mathrm{t}\rangle}: \exists x\left[x \in \text { boy }_{\varrho}\right.\right. \\
& \left.\left.\wedge Q=\left\{p \in D_{\langle\mathrm{s}, \mathrm{t}\rangle}: \exists y\left[y \in \text { girl }_{@} \wedge p=\left\{w^{\prime} \in D_{s}: x \text { admires }_{w^{\prime}} y\right\}\right]\right\}\right]\right\} \\
& =\left\{\begin{array}{l}
\left\{p \in D_{\langle\mathrm{s}, \mathrm{t}\rangle}: \exists y\left[y \in \operatorname{girl}_{@} \wedge p=\left\{w^{\prime} \in D_{s}: \text { Jeff admires }_{w^{\prime}} y\right\}\right]\right\}, \\
\left.p \in D_{\langle\mathrm{s}, \mathrm{t}\rangle}: \exists y\left[y \in \operatorname{girl}_{@} \wedge p=\left\{w^{\prime} \in D_{s}: \text { Abed admires }_{w^{\prime}} y\right\}\right]\right\}, \\
\left.p \in D_{\langle\mathrm{s}, \mathrm{t}\rangle}: \exists y\left[y \in \operatorname{girl}_{@} \wedge p=\left\{w^{\prime} \in D_{s}: \text { Troy admires }_{w^{\prime}} y\right\}\right]\right\}
\end{array}\right\}
\end{aligned}
$$

${ }^{9}$ It is possible to provide a recursive definition of pluralization that encompasses both $*$ and $* *$. We therefore speculate that $*$ and $* *$ are manifestations of the same abstract operation. We sketch an recursive definition for the pluralization operator $\mathfrak{P}$ below. We leave a detailed formal treatment to future work.

(i) $\mathfrak{P}\left(A_{\langle\sigma, \iota\rangle}\right)$

a. $=\varnothing(A)-\emptyset$

b. $\left.=\left\{\begin{aligned}<X_{1}, \ldots, X_{n}>: X_{1} & \in \mathfrak{P}\left\{x: \exists y \in X_{2}, \ldots, \exists z \in X_{n}[<x, y, \ldots, z>\in A]\right\} \\ \cdots & \wedge X_{n} \in \mathfrak{P}\left\{x: \exists y \in X_{1}, \ldots, \exists z \in X_{n-1}[<y, \ldots, z, x>\in A]\right\}\end{aligned}\right\} \quad \begin{array}{c}\iota=\mathrm{t} \\ \end{array}\right\}$

$A$ is a set of tuples

${ }^{10}$ Since an adequate semantics for questions requires intensionality, we assume that predicates are interpreted relative to a world parameter from here on. To simplify, we assume that NP restrictors are interpreted de re. We signify the actual world with the @ symbol.

${ }^{11}$ See Dayal (1996) and Fox (2012) for discussion of how the presuppositions of multiple questions motivated a more structured denotation. 
Distributivity and cumulativity in questions We observe that embedded multiple questions give rise to cumulative readings with a plural subject, as shown by the interpretation of (28).

(28) The three detectives have found out or are investigating which gang controls which estate. $\vDash$ for each detective, there is a sub-question that he has either found out the answer to, or is still investigating, and for each sub-question, there is a detective who has either found out the answer to it, or is still investigating it.

This phrasal cumulative reading is derived by having the $* *$-operator scope over the disjunction, as illustrated by the LF in (29). We provide a sample derivation illustrating how this account predicts the acceptability of the sentence in the mixed scenario in (30).

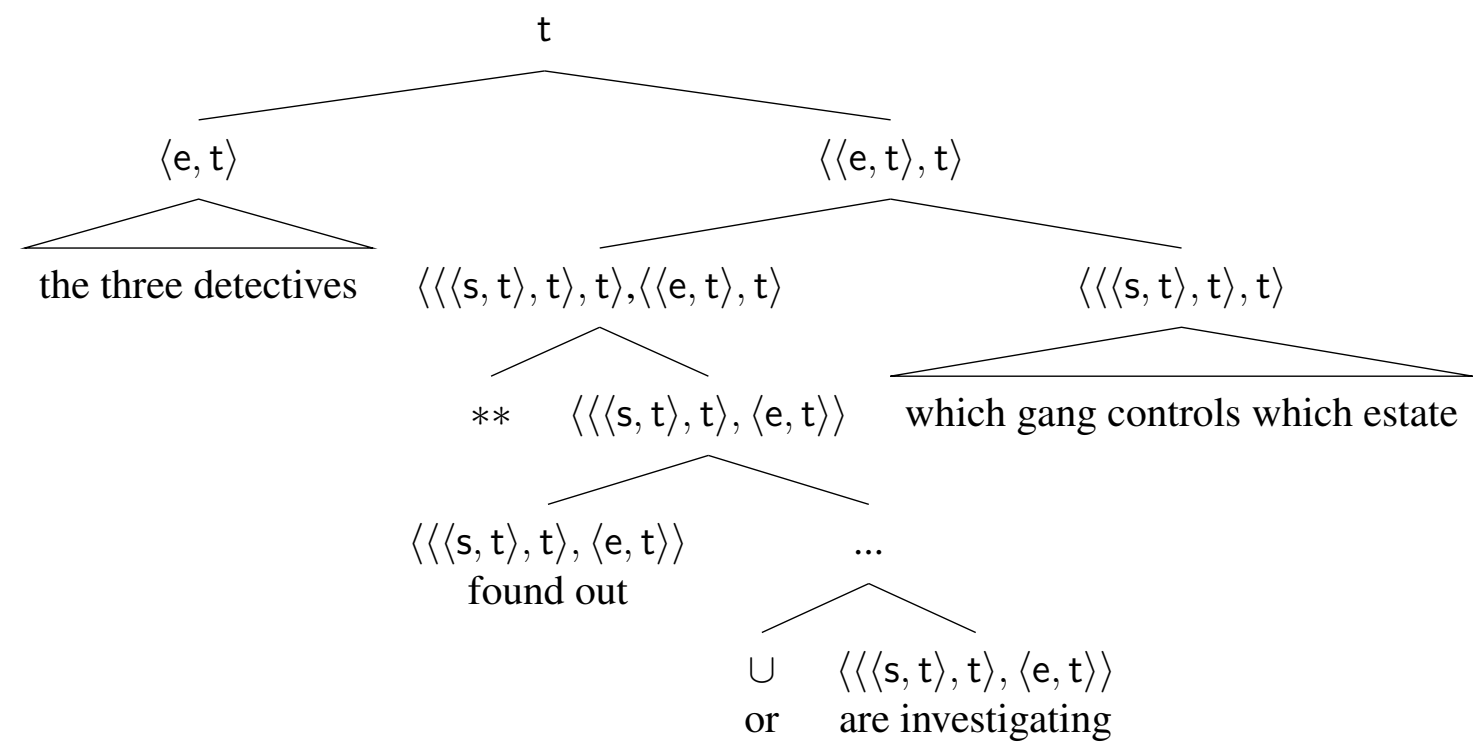

(30) a. $\quad$ the detectives $\rrbracket=\left\{d_{1}, d_{2}, d_{3}\right\}$

b. $\quad$ which gang controls which estate $\rrbracket=\left\{\begin{array}{l}Q_{1}=\text { which estate does } \mathrm{g}_{1} \text { control? } \\ Q_{2}=\text { which estate does } \mathrm{g}_{2} \text { control?, } \\ Q_{3}=\text { which estate does } \mathrm{g}_{3} \text { control? }\end{array}\right\}$

c. $\llbracket$ found out $\rrbracket=\left\{<\mathrm{d}_{1}, Q_{1}>\right\}$

d. 【are investigating $\rrbracket=\left\{<\mathrm{d}_{2}, Q_{2}>,<\mathrm{d}_{3}, Q_{3}>\right\}$

e. $\quad$ ffound out or are investigating $\rrbracket=\left\{<\mathrm{d}_{1}, Q_{1}>,<\mathrm{d}_{2}, Q_{2}>,<\mathrm{d}_{3}, Q_{3}>\right\}$

f. $\quad * *(\llbracket$ found out or are investigating $\rrbracket)$

$$
=\left\{\begin{array}{c}
<\left\{\mathrm{d}_{1}\right\},\left\{Q_{1}\right\}>,<\left\{\mathrm{d}_{2}\right\},\left\{Q_{2}\right\}>,<\left\{\mathrm{d}_{3}\right\},\left\{Q_{3}\right\}> \\
<\left\{\mathrm{d}_{1}, \mathrm{~d}_{2}\right\},\left\{Q_{1}, Q_{2}\right\}>,<\left\{\mathrm{d}_{1}, \mathrm{~d}_{3}\right\},\left\{Q_{1}, Q_{3}\right\}>, \\
<\left\{\mathrm{d}_{2}, \mathrm{~d}_{3}\right\},\left\{Q_{2}, Q_{3}\right\}> \\
<\left\{\mathrm{d}_{1}, \mathrm{~d}_{2}, \mathrm{~d}_{3}\right\},\left\{Q_{1}, Q_{2}, Q_{3}\right\}>
\end{array}\right\}
$$

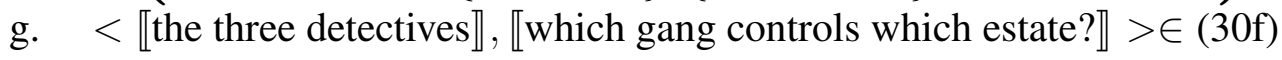

Phrasal distributivity, on the other hand, is not as readily available with embedded questions, as evidenced by the example in (31). Note that here we use scopal interaction with the quantificational DP at least two factors as a diagnostic for phrasal distributivity. Furthermore, note that 
unlike in our examples intended to test for cumulative readings with multiple questions such as (28), in (31) the predicate depends on takes the multiple question as its subject. This is because, as pointed out by de Vries (2015), inverse scope distributive readings are typically difficult to retrieve in English. The $*$-operator must take scope above the quantificational DP at least two factors in order to derive the putative reading where the two factors co-vary with sub-questions of which boy likes which girl, a reading which we claim is not available in English.

(31) Which girl likes which boy depends on at least two factors.

$\not \models$ which boy Annie likes depends on at least two factors, which boy Britta likes depends on at least two factors, etc.

In order to account for this apparent discrepancy, we propose the following negative licensing condition on the insertion of the $*$ and $* *$-operators:

\section{Condition on phrasal plurality $(* / * *)$}

The $*$ and $* *$-operators may freely attach to any extended projection unless that projection is morphosyntactically singular.

This negative licensing condition correctly rules out phrasal distributivity with interrogatives in subject position since questions, even when they denote pluralities, do not impose (and in fact resist) plural marking on the verb. Note that this condition does not rule out phrasal cumulativity with interrogatives in object position, (28), since in English objects do not agree with verbs for number.

6. Predictions and open questions. There are certain cases where questions do trigger plural agreement. Specifically, in British English coordinated interrogatives may trigger either singular or plural agreement. When the marking is plural, as in (33), phrasal distributivity becomes available, as evidenced by the availability of the interpretation below.

(33) Which boy likes Mary and which boy likes Sue depend(s) on at least 2 factors.

$\vDash$ which boy likes Mary depends on at least two factors, and which boy likes Sue depends on at least two factors. (only with plural agreement)

In principle, our licensing condition on $* / * *$ rules out phrasal distributivity when the plural interrogative is in the object position, if the VP is singular. This is difficult to test in English since inverse scope distributive readings are generally difficult to retrieve, but a putative test case would be the example in (34). We leave further investigation of this question to future work.

(34) John found out or is still investigating which gang controls which estate.

Similarly, we predict no phrasal cumulativity with interrogatives in subject position, given the singular marking on the verb. We give a putative test case in (35), although we find the judgements to be not so clear. Concretely, we expect (35) to be false in a mixed scenario, where, for example, there are three students $s_{1}, s_{2}$ and $s_{3}$, and three professors $p_{1}, p_{2}$ and $p_{3}$, which class $s_{1}$ aced is of interest to $p_{1}$ which class $s_{2}$ aced is irrelevant to $p_{2}$, and which class $s_{3}$ aced is irrelevant to $p_{3}$.

(35) Which student aced which class is of interest to or is irrelevant to the three professors.

7. Conclusion. In this short paper, we have attempted to sketch an account of (a) the domain generality of semantic plurality, by adopting a framework wherein distributive and cumulative inferences are captured via type-flexible operators, and (b) the constrainedness of semantic plurality, 
by arguing that the presence of these operators $(*$ and $* *)$ is blocked by morphosyntactic singularity. We have presented evidence for this picture on the basis of group nouns and plural agreement in British English, as well as by investigating the behavior of semantically plural interrogatives. If semantic plurality really is domain general, as we claim, we expect to find plurality effects in other domains as well, such as events, times, etc. We leave a more thorough investigation into the pervasiveness of semantic plurality to future work.

\section{References}

Barker, Chris. 1992. group terms in English: representing groups as atoms. Journal of Semantics 9. 69-93.

Beck, Sigrid \& Uli Sauerland. 2000. Cumulation is needed: A reply to winter (2000). Natural Language Semantics 8(4). 349-371. doi:10.1023/A:1011240827230. https:// link. springer.com/article/10.1023/A:1011240827230.

Beck, Sigrid \& Yael Sharvit. 2002. Pluralities of questions. Journal of Semantics 19(2). 105-157. doi:10.1093/jos/19.2.105. http://jos.oxfordjournals.org/content/19/2/ 105.

Champollion, Lucas. 2015. Ten men and women got married today: Noun coordination and the intersective theory of conjunction. Journal of Semantics ffv008. doi:10.1093/jos/ffv008. http://jos.oxfordjournals.org/lookup/doi/10.1093/jos/ffv008.

Dayal, Veneeta. 1996. Locality in WH quantification, vol. 62 Studies in Linguistics and Philosophy. Springer Netherlands. http://link.springer.com/10.1007/ 978-94-011-4808-5.

de Vries, Hannah. 2015. Shifting sets, hidden atoms : the semantics of distributivity, plurality and animacy: Utrecht University dissertation. http: / / dspace.library.uu.nl/ handle/1874/312186.

Elbourne, Paul. 1999. Some correlations between semantic plurality and quantifier scope. In NELS 29: Proceedings of the North East Linguistic Society, .

Fox, Danny. 2012. The semantics of questions. Class notes, MIT seminar.

Hamblin, Charles L. 1973. Questions in montague english. Foundations of Language 10(1). 41-53.

Heim, Irene \& Angelika Kratzer. 1998. Semantics in generative grammar (Blackwell textbooks in linguistics 13). Blackwell.

Karttunen, Lauri. 1977. Syntax and semantics of questions. Linguistics and Philosophy 1(1). 3-44. doi:10.1007/BF00351935. http://link.springer.com/article/10. 1007 / BF 00351935.

Kotek, Hadas. 2014. Composing questions: Massachusetts Institute of Technology dissertation.

Landman, Fred. 1996. Plurality. In Shalom Lappin (ed.), The handbook of contemporary semantic theory, 425-458. Blackwell.

Link, Godehard. 1983. The logical analysis of plurals and mass terms: A lattice-theoretic approach. In P. Portner and B. H. Partee (ed.), Formal semantics - the essential readings, 127-147. Blackwell. 
Munn, Alan. 1999. First conjunct agreement: Against a clausal analysis. Linguistic Inquiry 30(4). 643-668. doi:10.1162/002438999554246. http://dx.doi.org/10.1162/ 002438999554246.

Nicolae, Andreea Cristina. 2013. Any questions? polarity as a window into the structure of questions: Harvard University dissertation.

Partee, Barbara. 1986. Noun-phrase interpretation and type-shifting principles. In J. Groenendijk, D. de Jongh \& M. Stokhof (eds.), Studies in discourse representation theory and the theory of generalized quantifiers, 115-143. Dordrecht: Foris.

Pearson, Hazel. 2011. A new semantics for group nouns. In Mary Byram Washburn, Katherine McKinney-Bock, Erika Varis, Ann Sawyer \& Barbara Tomaszewicz (eds.), Proceedings of the $28^{\text {th }}$ West Coast Conference on Formal Linguistics, 160-168. Cascadilla Proceedings Project.

Sauerland, Uli \& Paul Elbourne. 2002. Total reconstruction, PF movement, and derivational order. Linguistic Inquiry 33(2). 283-319. doi:10.1162/002438902317406722. http://www . mitpressjournals.org/doi/10.1162/002438902317406722.

Scha, Remko. 1981. Distributive, collective and cumulative quantification. In J. A. G. Groenendijk, T. M. V. Janssen \& M. B. J. Stokhof (eds.), Formal methods in the study of language, part 2, 483-512. Mathematisch Centrum.

Schwarzschild, Roger. 1996. Pluralities, vol. 61 Studies in Linguistics and Philosophy. Springer Netherlands. http://link.springer.com/10.1007/978-94-017-2704-4.

Winter, Yoad. 2001. Flexibility principles in boolean semantics: the interpretation of coordination, plurality, and scope in natural language (Current studies in linguistics 37). MIT Press. 